\title{
Measurement of turbulent flow in a narrow open channel
}

\author{
Sankar Sarkar \\ Physics and Applied Mathematics Unit, Indian Statistical Institute Kolkata, West Bengal 700108, India. \\ Tel.: +91 332575 3026. Fax: +91 332577 3026. E-mail: sankar79_v@isical.ac.in
}

\begin{abstract}
The paper presents the experimental results of turbulent flow over hydraulically smooth and rough beds. Experiments were conducted in a rectangular flume under the aspect ratio $b / h=2(b=$ width of the channel $0.5 \mathrm{~m}$, and $h=$ flow depth $0.25 \mathrm{~m}$ ) for both the bed conditions. For the hydraulically rough bed, the roughness was created by using $3 / 8^{\prime \prime}$ commercially available angular crushed stone chips; whereas sand of a median diameter $d_{50}=1.9 \mathrm{~mm}$ was used as the bed material for hydraulically smooth bed. The three-dimensional velocity components were captured by using a Vectrino (an acoustic Doppler velocimeter). The study focuses mainly on the turbulent characteristics within the dip that were observed towards the sidewall (corner) of the channel where the maximum velocity occurs below the free-surface. It was also observed that the nondimensional Reynolds shear stress changes its sign from positive to negative within the dip. The quadrant plots for the turbulent bursting shows that the signs of all the bursting events change within the dip. Below the dip, the probability of the occurrence of sweeps and ejections are more than that of inward and outward interactions. On the other hand, within the dip, the probability of the occurrence of the outward and inward interactions is more than that of sweeps and ejections.
\end{abstract}

Keywords: Flow measurement; Turbulent flow; Dip-phenomenon; Turbulent bursting; Open channel flow.

\section{INTRODUCTION}

Turbulent flow in open channel is a matter of common interest to the researchers of many fields, e.g., hydraulics, hydromechanics, fluid mechanics, applied mathematics, physics, environmental engineering, and so on. Due to the spatial and temporal heterogeneity in the flow characteristics, open channel turbulence is an interesting topic to the researchers till date. With the advancement of the measuring techniques and devices, numerous research works have been carried out experimentally and theoretically in open channel turbulence. Measurements of the turbulent parameters are generally performed by laser Doppler anemometry, acoustic Doppler velocimeter, acoustic Doppler velocity profiler, hot wire anemometry, laser ultrasonic Doppler velocimetry, and particle imaging velocimetry, etc. It is experienced by many researchers that in spite of taking enough precautions, the velocity measuring devices show some degree of uncertainness while capturing the velocity data. This may be the reason for what researchers have some dissatisfaction with different velocity measuring devices. The captured data sometimes get influenced by spurious data those are called as spikes. Before using the captured data to analyze for turbulent characteristics they should be free from spikes. Goring and Nikora (2002) is probably the most popular paper that provides the details of despiking the ADV data where several despiking algorithms are available. Later, some of other algorithms have been developed by Cea et al. (2007), Parsheh et al. (2010), Wright and Baas (2013), and MacVicar et al. (2014) etc.

According to Chow (1959) and Dey (2014), open channels can be classified as wide and narrow based on their aspect ratio $b / h$. For $b / h<5$, the channel is called as narrow channel and for $b / h>10$, the channel is known as wide channel. Chow (1959) observed that strong secondary currents are developed in the narrow channels, where the maximum velocity takes place below the free surface involving a deviation of the velocity profile from the log-wake law, which is known the dip phenomenon. On the other hand, Tracy and Lester (1961) divided an open channel into two parts, namely, the central region and the corner region of flow while measured with respect to the width. Auel et al. (2014) argued that the dip phenomenon is observed at the corner regions and their effects diminish toward the flume centre. They observed that the effect of Froude number is less prominent on the flow characteristics in comparison with that of aspect ratio. They also argued that the streamwise turbulent intensities in the flume centre are almost independent of the Froude number in case of wide open channel but in case of narrow channels, they show different patterns at different Froude numbers. An increase in the vertical turbulence intensity was observed by them with an increase in Froude number for wide open channel. The streamwise turbulence intensity and Reynolds shear stress deviate from the expected linear trend in case of $b / h<3$, than those with higher values of $b / h$. Vanoni (1941) also suggested that the dip phenomenon is observed at the corner zones even for the wide open channels where the velocity profile deviates from the log-wake law. Absi (2011) suggested an ordinary differential equation for velocity distribution and dip-phenomenon in open channel flows. Rodríguez and García (2008) studied the effect of secondary flow occurred in the corner region of a narrow open channel. Sarma et al. (2000) proposed a binary law for the velocity distribution in an open channel which is a combination of the logarithmic law for the inner region and the parabolic law for the outer region. Guo and Julien (2008) proposed a modified log-wake law to fit the velocity distribution for flow with dip-phenomenon. Yang et al. (2004) proposed a methodology to estimate the Reynolds shear stress distribution and velocity dip phenomenon in a smooth open channel. They proposed a modified log-law to study the dip phenomenon based on the analysis of the RANS equations. This law involves two logarithmic distances, one from the bed and the other from the free surface using a parameter named as for dip-correction factor.

To observe the flow over a rough bed experimentally, gravel, sand, sand-gravel mixture, crushed stone, artificial vegetation, glass breeds are generally used. It is important to mention here that roughness elements with different shapes and sizes have different properties and therefore their presence make differences in the flow characteristics. For an instance, depend- 
ing on the velocity profiles and shape of the roughness elements, Franca and Lemmin (2009), and Mignot et al. (2009) classified the rough beds into different classes as log, S-shaped, and accelerated classes. Although their effects may not be so visible with small roughness, larger size of the roughness and compactness of the roughness materials make the differences. Rodríguez and García (2008) used crushed stone chips of $d_{90}=$ $10 \mathrm{~mm}$ to study the effect of secondary flow with aspect ratios of 8.5 and 6.3, respectively flow in a straight laboratory flume. Najafabadi et al. (2015) have done an experimental study of turbulent flow in a gravel-bed channel with vegetated walls and showed that sweeps and ejections dominate near the vegetated flume walls and found no negative shear stress near the water surface for favorable pressure gradient flows.

Although according to the present scenario, researchers have a considerable understanding in open channel flow, some issues are yet to explore. For an instance, the turbulent bursting has not been thoroughly studied within the dip observed in an open channel flow. Although a good number of experimental and theoretical works have been done to study the velocity and stress distribution for dip phenomenon, but no further progress have been made on this issue. One of the main causes is that the turbulent events like turbulent kinetic energy, energy flux and turbulent bursting show significant changes within the shearlayer only. On the other hand, above the shear-layer, their behaviors are almost invariable with an increase in depth for a plane rough or smooth open channel flow.

The present study is therefore undertaken to observe the characteristics of turbulent flow over a hydraulically smooth and a rough bed within the dip.

\section{EXPERIMENTAL FACILITY}

Two sets of experiments were carried out at the Fluvial Mechanics laboratory of the Indian Statistical Institute Kolkata,
India. The rectangular flume used for the experiment is $0.5 \mathrm{~m}$ wide, $0.5 \mathrm{~m}$ deep and $20 \mathrm{~m}$ long. The flume has Perspex sidewalls that facilitate the observation of the flow. Uniform streamwise bed slopes were maintained in the experiments using uniformly graded sand of $d_{50}=1.9 \mathrm{~mm}$ and $3 / 8^{\prime \prime}$ commercially available angular crushed stone chips. For hydraulically smooth bed, the Nikuradse's roughness length $k_{s}$ was considered as the median size $\left(d_{50}\right)$ of the sand. On the other hand, the value of $k_{s}$ for hydraulically rough bed was measured from the bed fluctuations as $36 \mathrm{~mm}$. For both the runs, flow depth was $0.25 \mathrm{~m}$ above the bed level $(z / h=0)$. Bed-slope and flow depths along the centerline of the flume were measured by a Vernier point gauge with a precision of $\pm 0.1 \mathrm{~mm}$. After preparing the bed, the required discharge was applied by a pump. At least half an hour time was given to ensure the stability of the flow before the actual velocity measurement. The measuring locations were chosen from the centerline of the flume towards the sidewall (corner). The $x-, y$-, and $z$-axes are considered in the streamwise, lateral and vertical directions, respectively. The origin of the $y$-axis was located at the centerline of the flume where $y /(0.5 b)=0$ and its values were taken positive towards the sidewall of the flume. Nine locations were chosen for the data capturing. However, vertical distributions for five locations are presented herein to avoid the data congestion. The measuring locations presented here were taken with respect to the one-half of the width of the flume. These locations are: $y /(0.5 b)=0,0.2,0.4,0.6$, and 0.8 , respectively. All the measuring locations were at a distance of $8.5 \mathrm{~m}$ from the upstream of the flume ensuring that the measurements were taken in the fully developed zone of turbulence. Details of the experimental parameters are tabulated in Table 1 and the schematic of the experimental setup is shown in Fig. 1.

Table 1. Experimental parameters.

\begin{tabular}{ccccccccccc}
\hline Set & $k_{s}(\mathrm{~mm})$ & $S$ & $b / h$ & $U$ & $F r$ & $R e$ & $u_{*_{1}}$, slope & $u_{* 2}$, vel. & $u_{* 3}$, stress & $R *$ \\
\hline 1 & 1.9 & 0.00033 & 2 & 0.44 & 0.28 & 445127 & 0.029 & 0.029 & 0.024 & 55 \\
2 & 36 & 0.003 & 2 & 0.96 & 0.65 & 958531 & 0.086 & 0.0857 & 0.078 & 3096 \\
\hline
\end{tabular}

$S=$ the bed slope, $b=$ width of the channel, $h=$ the flow depth, $U=$ the depth-averaged velocity, $F r=$ Froude number $(=U / \sqrt{g h}), R e=$ Reynolds number $(4 U h / v), v=$ kinematic viscosity of water, $u_{*_{1}}, u_{*_{2}}$, and $u_{*_{3}}=$ shear velocities obtained from the slope, velocity profiles and Reynolds shear stress distributions, respectively, and $R_{*}=$ particle Reynolds number $\left(=u_{* 1} k_{s} / v\right)$.

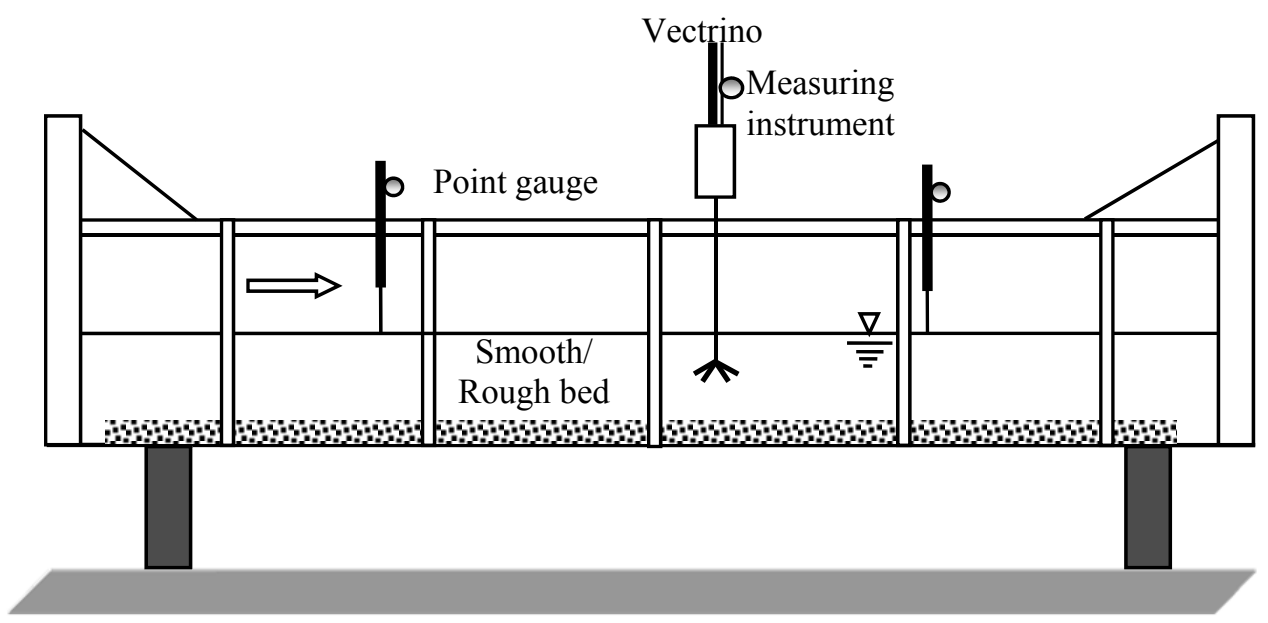

Fig. 1. Schematic of experimental set-up. 


\section{DATA CAPTURING AND FILTERING OF RAW DATA}

Three-dimensional water velocities were captured using a 4beam Vectrino. The Vectrino is an acoustic Doppler velocimeter manufactured by Nortek. It was operated with an acoustic frequency of $10 \mathrm{MHz}$ and a sampling rate of $100 \mathrm{~Hz}$. Although the sampling rate can be extended up to $200 \mathrm{~Hz}$, it was experienced that above $100 \mathrm{~Hz}$ the Vectrnio data are prone to more spikes. Four acoustic beams of the Vectrino meet at a distance of $50 \mathrm{~mm}$ below the probe head that form a vertical cylinder with sampling volume of $6 \mathrm{~mm}$ diameter and $1-15 \mathrm{~mm}$ adjustable height. The measuring location was $50 \mathrm{~mm}$ below the probe and therefore the measurement was not possible for the top 50 $\mathrm{mm}$ flow depth. Before conducting the actual experiment, some trials were taken to estimate the uncertainty in the measurement which suggested that the sampling duration for $300 \mathrm{~s}$ over a single point is sufficient to get time-independent velocity components. Up to the vertical distance $20 \mathrm{~mm}$ above the bed level, the lowest sampling height of the Vectrino measurements was used as $1 \mathrm{~mm}$ considering the importance of the near-bed profile. The lowest measuring point was $2 \mathrm{~mm}$ above the bed level. In general, the Vectrino signal correlation values greater than $70 \%$ was used as the minimum. However, nearest the bed, the range of the Vectrino signal correlation sometimes drops up to $65 \%$. Throughout the experiments, the minimum SNR (signalto-noise ratio) was maintained as 17 . Both the runs were conducted under subcritical flow conditions. During the experiment, the beds were immobile as no movement of the bed materials was observed. The raw Vectrino data were despiked by the acceleration threshold method (Goring and Nikora, 2002), which was capable in identifying and substituting spikes. The threshold values were chosen in the ranges between $1-1.5$ by trial and error (Dey and Das, 2012).

\section{RESULTS AND DISCUSSION}

Vertical distributions of time-averaged streamwise velocity and Reynolds shear stress

Figs. 2(a-b) show the vertical variations of nondimensional time-averaged streamwise velocity in flows with different bed conditions: hydraulically smooth and rough beds (set 1 and set 2 , respectively). The streamwise velocities were nondimensionalised by the shear velocity that fit the logarithmic law of velocity distributions, i.e., $u / u_{*}=(1 / \kappa) \ln \left(z / k_{s}\right)+5.5$ for hydrauli- cally smooth bed, and $u / u_{*}=(1 / \kappa) \ln \left(z / k_{s}\right)+8.5$ for hydraulically rough bed. Here, $u_{*}$ is the shear velocity and $\kappa$ is the von-Karman constant considered as 0.41 . To nondimensionalize the streamwise velocity, $u_{*_{2}}$ as shown in Table 1 was used. The vertical distance $z$ was nondimensionalized as $z / k_{s}$. It is shown in Table 1 that the shear velocities obtained from the velocity distribution is very close to the values of those obtained from the bed slope $\left(u_{*_{1}}=\sqrt{g h S}\right)$. For both the data sets, the nondimensional velocities agree well with the theoretical values for the lateral distances up to $y /(0.5 b) \leq 0.4$. On the other hand, for the lateral distances $y /(0.5 b)>0.4$, the nondimensional streamwise velocities are less than those of the theoretical values above $z / k_{s}=0.4(z / h \approx 0.6)$ for both Set 1 and Set 2 . For $y /(0.5 b)>0.4$, the velocity data above $z / k_{s}=0.4(z / h \approx 0.6)$ signify the velocity dip that are occurred by the secondary effect from the side-wall where the streamwise velocity attains the maximum value below the free-surface. This velocity-dip is responsible for the deviation of the streamwise velocity from the theoretical values. The type of flow achieved here is that of secondary motions in straight uniform open-channel flows and can also be called as 'turbulence-driven secondary currents' or 'secondary currents of Prandtl's second kind' (Nezu and Nakagawa, 1993).

The vertical variations of nondimensional Reynolds shear stress are depicted in the Figures 3(a) and 3(b) for hydraulically smooth and rough beds, respectively. The Reynolds shear stresses are nondimensionalised by the shear velocities that were obtained by extending the Reynolds shear stress profiles on to the bed level as $\tau_{0}=-\left.\rho \overline{u^{\prime} w^{\prime}}\right|_{z=0}$, where $\tau_{0}$ is the bed shear stress. The shear velocities were then calculated as $u_{*}=$ $\left(\tau_{0} / \rho\right)^{0.5}$. For the nondimensionalizing of Reynolds shear stress $u_{*_{3}}$ written in Table 1 is used. In general, the nondimensional Reynolds shear stress, $-\rho \overline{u^{\prime} w^{\prime}} / u_{*}^{2}$ for an open channel flow follows the traditional linear law. This linear law is also called as gravity law of Reynolds shear stress in uniform open channel flow. According to the linear law, nondimensional Reynolds shear stress at any depth is taken as $-\rho \overline{u^{\prime} w^{\prime}} / u_{*}^{2}=1-z / h$. According to the figures, no significant changes were observed in the profiles of nondimensional Reynolds shear stresses for flows with different bed conditions. All the nondimensional Reynolds shear stress data sets follow the gravity line for $y /(0.5 b)=\leq 0.6$ although for smooth bed, the $-\rho \overline{u^{\prime} w^{\prime}} / u_{*}^{2}$ deviates
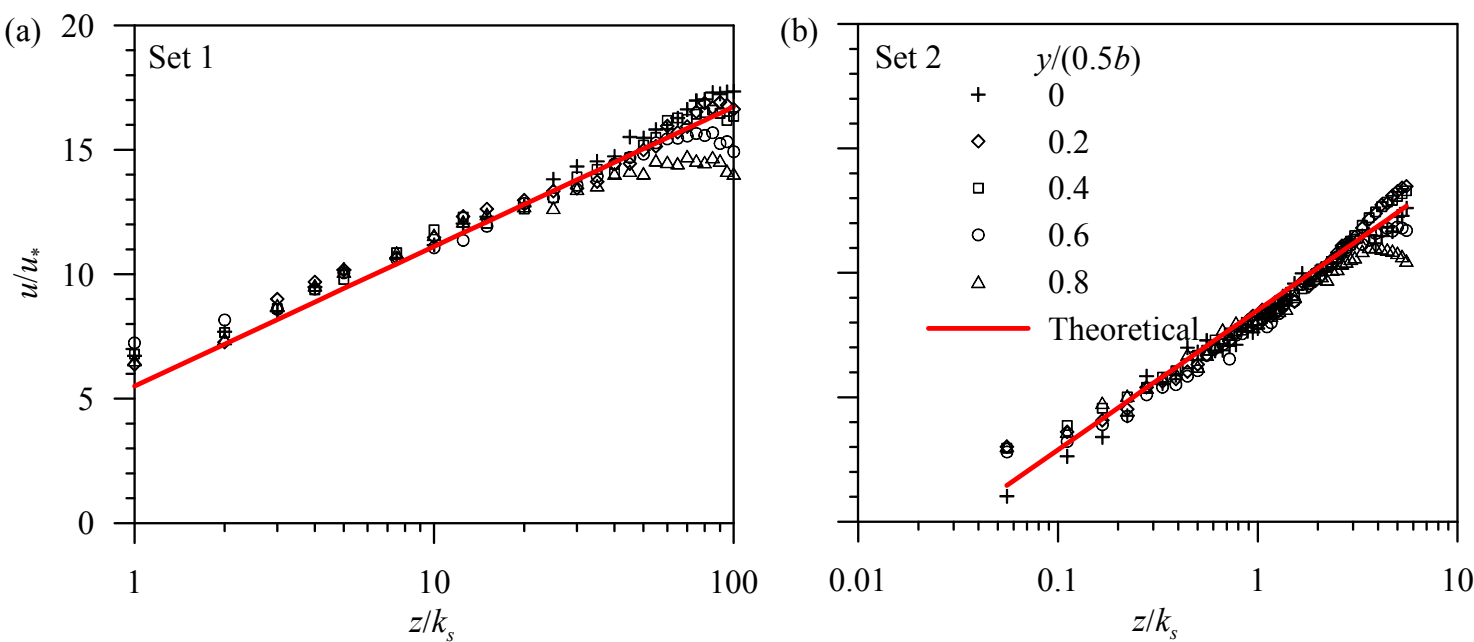

Fig. 2. Vertical distributions of the time-averaged streamwise velocity for (a) hydraulically smooth bed and (b) hydraulically rough bed. 

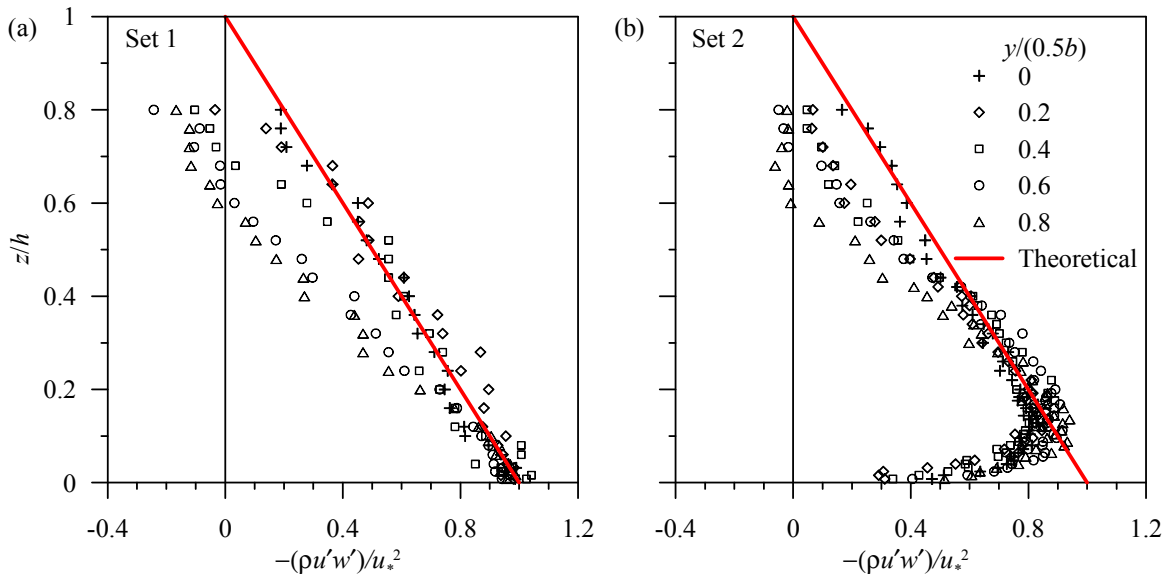

Fig. 3. Vertical distribution of nondimensional Reynolds shear stress for (a) hydraulically smooth and (b) hydraulically rough bed.
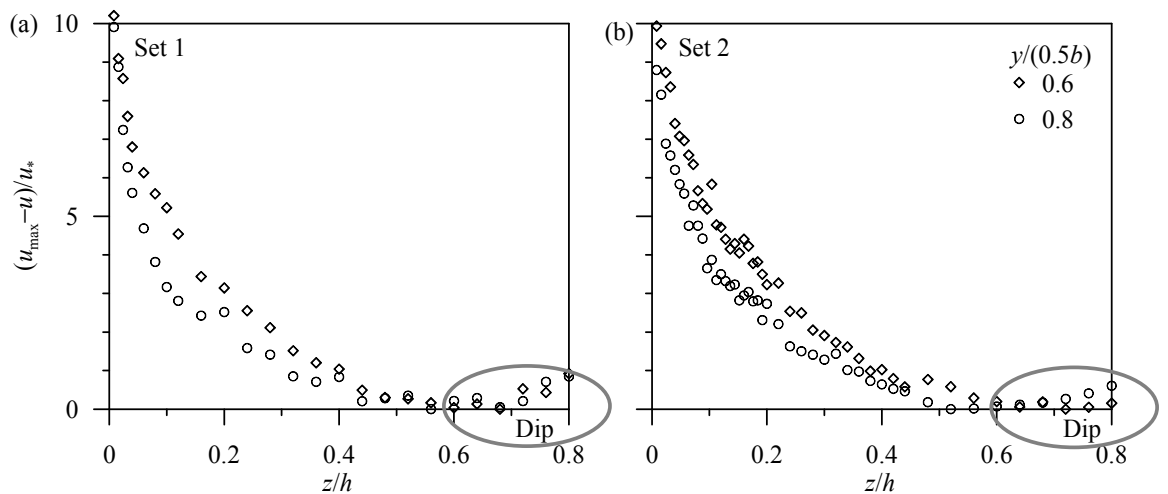

Fig. 4. Vertical distribution of nondimensional velocity defect for (a) hydraulically smooth and (b) hydraulically rough bed at $y /(0.5 b)=0.6$ and 0.8 .

more from the gravity line throughout the depth when they are measured at locations other than $y /(0.5 b)=0$. However, towards the corner of the sidewall, at the vertical distance above $z / h=0.6$, the Reynolds stress profiles deviate from the gravityline. For the locations $y /(0.5 b) \geq 0.6$, at $z / h \geq 0.6$, the nondimensional Reynolds shear stress deviates from the traditional linear law and its sign changes from positive to negative for both the hydraulically smooth and rough beds. This is in confirmation with Yang et al. (2004). The changes of sign of nondimensional Reynolds shear stress signify the existence of dip in open-channel flow.

Figs. 4(a) and 4(b) show the vertical distributions of nondimensional velocity defect for smooth and rough beds, respectively at $y /(0.5 b)=0.6$ and 0.8 . The velocity defect was calculated from the difference between maximum time-averaged velocity of the velocity profile and the time-averaged velocity at a certain depth. The velocity defect was nondimensionalised as $\left(u_{\max }-u\right) / u_{*_{2}}$. The velocity defect distributions indicate that the velocity is maximum (velocity defect is zero) at $z / h \approx 0.6$ and $y /(0.5 b)=0.6$ and 0.8 for both the smooth and rough beds and above this the velocity is less than the maximum velocity.

\section{TURBULENT BURSTING}

The flow pattern near the wall in a turbulent boundary layer is repetitive and follows a quasi-cyclic process, called as the bursting process (Corino and Brodkey, 1969; Grass, 1971; Kline et al., 1967). Close to the bed, the flow consists of an alternation of low-speed and high-speed regions, those are also called as low-speed and high-speed streaks. Similar to the sepa- ration of a boundary layer, a low-speed fluid streak becomes unstable due to a local and temporary adverse pressure gradient and enters into the main body of the flow. This is called as the ejection. The ejected fluid which remains as a result of retardation is brushed away by high-speed fluid towards the wall in a process called as the sweep. Two other events are known as outward interactions and inward interactions. However, ejections and sweeps are the predominating events in the turbulent bursting in general open-channel flow problems.

The quadrant analysis introduced by Willmarth and $\mathrm{Lu}$ (1972) is a useful tool to describe the bursting phenomena. According to the quadrant analysis, the local flow behavior is divided into four quadrants. Based on the signs of $u^{\prime}$ and $w^{\prime}$, the events of the quadrants are classified as: $Q_{1}$ events called as outward interactions formed in first-quadrant, where $u^{\prime}>0$ and $w^{\prime}>0 ; Q_{2}$ events called as ejections in second-quadrant, where $u^{\prime}<0$ and $w^{\prime}>0 ; Q_{3}$ events called as inward interactions formed in third-quadrant, where $u^{\prime}<0$ and $w^{\prime}<0$; and $Q_{4}$ events called as sweeps in fourth-quadrant, where $u^{\prime}>0$ and $w^{\prime}<0$.

A hole-size parameter $H$, determined by the curve $\left|u^{\prime} w^{\prime}\right|=$ $H\left(u_{r m s}^{\prime} \times w_{r m s}^{\prime}\right)$ is used to differentiate the fractional contributions to $-\overline{u^{\prime} w^{\prime}}$ at four different quadrants. In order to take all the data pairs $\left(u^{\prime}, w^{\prime}\right)$ into consideration, the hole-size $H=0$ is used. The contributions to total Reynolds shear stress from the quadrant $i$ outside the hole region of size $H$ is estimated by

${\overline{u^{\prime} w^{\prime}}}^{i, H}=\underset{T \rightarrow \infty}{L t} \frac{1}{T} \int_{0}^{T} u^{\prime}(t) w^{\prime}(t) \lambda_{i, H}(z, t) d t$ 
where $T$ is the time of sampling and $\lambda_{i, H}(t)$ is the detection function given by $\lambda_{i, H}(t)=1$, if $\left(u^{\prime}, w^{\prime}\right)$ is in quadrant $i$ and if $\left|u^{\prime} w^{\prime}\right| \geq H\left(\overline{u^{\prime} u^{\prime}}\right)^{0.5}\left(\overline{w^{\prime} w^{\prime}}\right)^{0.5}$, and $\lambda_{i, H}(t)=0$, otherwise. The fractional contributions of conditional Reynolds shear stress can be calculated by

$$
\langle R S\rangle_{i, H}={\overline{u^{\prime} w^{i}}}^{i, H} /{\overline{u^{\prime} w^{\prime}}}^{\prime}
$$

The algebraic summation of contributions from different bursting events for $H=0$ is therefore unity, that is

$$
\langle R S\rangle_{1,0}+\langle R S\rangle_{2,0}+\langle R S\rangle_{3,0}+\langle R S\rangle_{4,0}=1 .
$$

The probability of occurrence of the bursting events $P_{i, H}$ can be calculated by Eq. (3) (Dey and Nath, 2010) as:

$$
P_{i, H}=\frac{\int_{t=0}^{t=T} \lambda_{i, H}(z, t) d t}{\int_{t=0}^{t=T}\left[\lambda_{1, H}(z, t)+\lambda_{2, H}(z, t)+\lambda_{3, H}(z, t)+\lambda_{4, H}(z, t)\right] d t}
$$

The algebraic summation of occurrence of the bursting events for $H=0$ is unity, that is $P_{1,0}+P_{2,0}+P_{3,0}+P_{4,0}=1$. The ratio of sweep and ejection is written as $\langle R S\rangle_{r}=$ $\langle R S\rangle_{4} /\langle R S\rangle_{2}$.
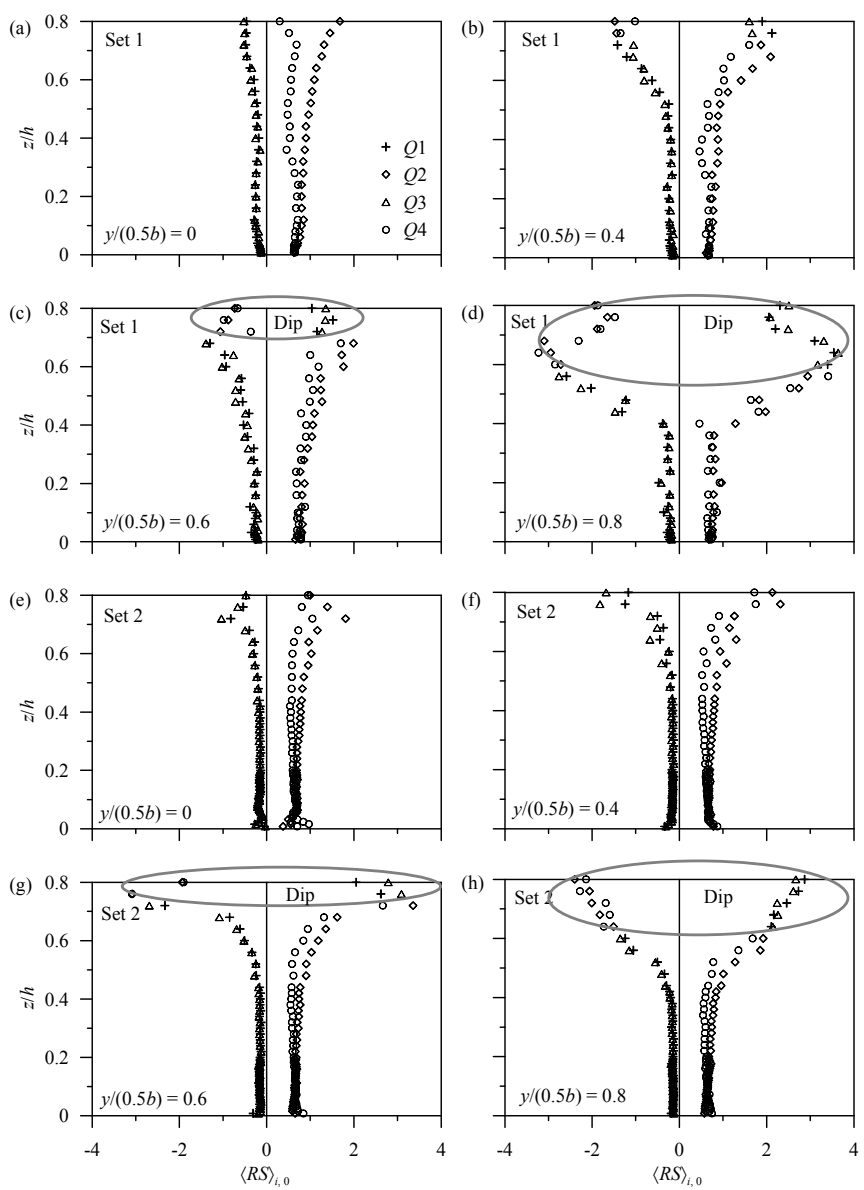

Fig. 5. Vertical distribution of conditional Reynolds shear stress $\langle R S\rangle_{i, H}$ for $H=0$ for $(\mathrm{a}-\mathrm{d})$ hydraulically smooth and $(\mathrm{e}-\mathrm{h})$ hydraulically rough bed at $y /(0.5 b)=0,0.4,0.6$ and 0.8 .
The vertical variations of $\langle R S\rangle_{i, H}$ for $H=0$ are shown in Figs. $5(\mathrm{a}-\mathrm{d})$ for smooth bed and Figs. 5(e-h) for rough bed. The quadrant plots for smooth bed indicate that close to the bed, sweeps and ejections start with almost same values. But, as the vertical distance increases, ejections become the predominating event throughout the depth for the $y /(0.5 b)<0.6$. On the other hand inward and outward interactions remain insignificant throughout the depth for $y /(0.5 b)<0.6$.

The quadrant plots for the rough bed show that close to the bed, sweeps become the most dominating events and with increase in vertical distance ejections become predominating for the data set $y /(0.5 b)<0.6$. Inward and outwards interactions remain insignificant throughout the depth for the data set $y /(0.5 b)<0.6$.

However, for both the smooth and rough beds, at $y /(0.5 b) \geq$ 0.6 and $z / h \approx 0.6$, the signs of the bursting events change which indicate the negative values of nondimensional Reynolds shear stress within the dip. Below the dip, their profiles are similar to that of bursting plots for $y /(0.5 b)<0.6$. The vertical variations of $\langle R S\rangle_{i, H}$ for $H=2$ are shown in Figs. 6(a-d) for smooth bed and Figs. $6(\mathrm{e}-\mathrm{h})$ for rough bed. Although, their values are different from $\langle R S\rangle_{i, H}$ for $H=0$, the overall behavior are similar.

The probability of the occurrence of the bursting events $P_{i, H}$ are shown in Figs. 7(a-d) for smooth bed and Figs. 7(e-h) for rough bed for the hole size, $H=0$. It is evident from the figures that close to the bed, probability of occurring sweeps and
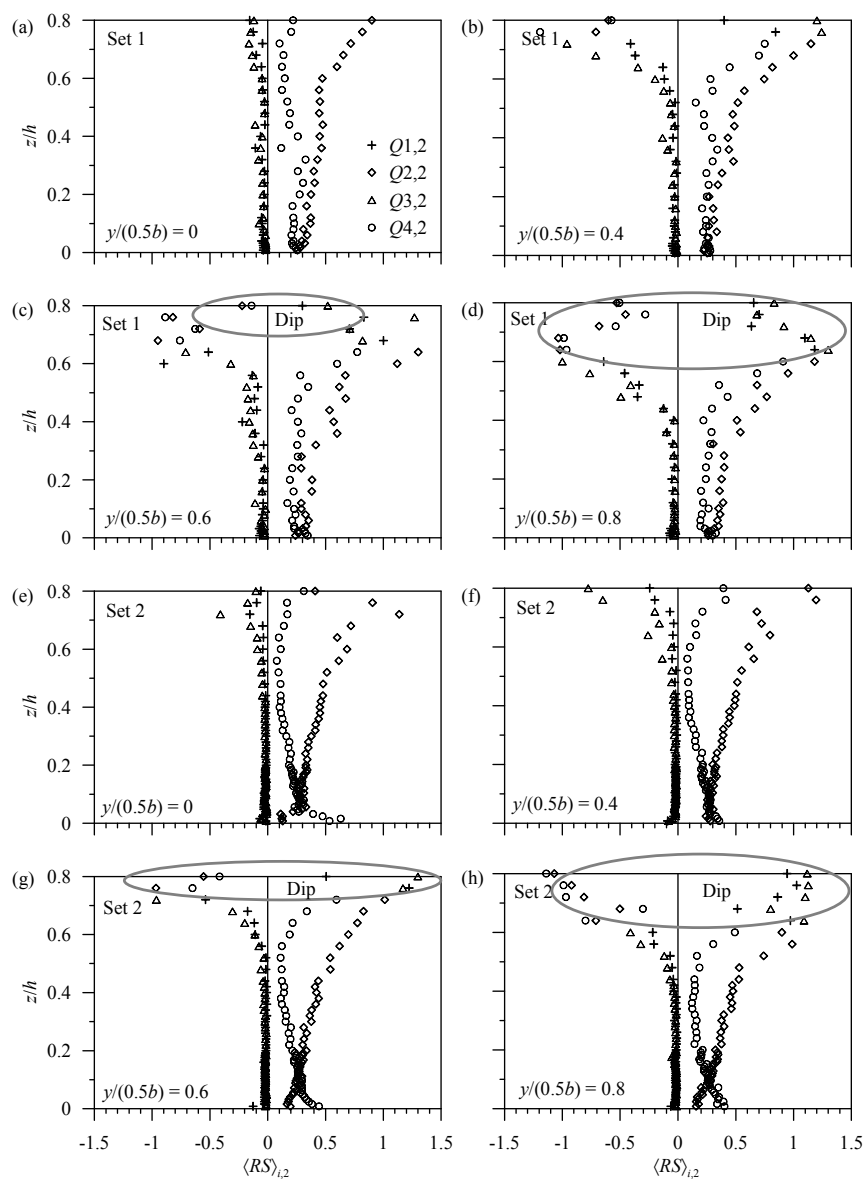

Fig. 6. Vertical distribution of conditional Reynolds shear stress $\langle R S\rangle_{i, H}$ for $H=2$ for (a-d) hydraulically smooth and (e-h) hydraulically rough bed at $y /(0.5 b)=0,0.4,0.6$ and 0.8 . 

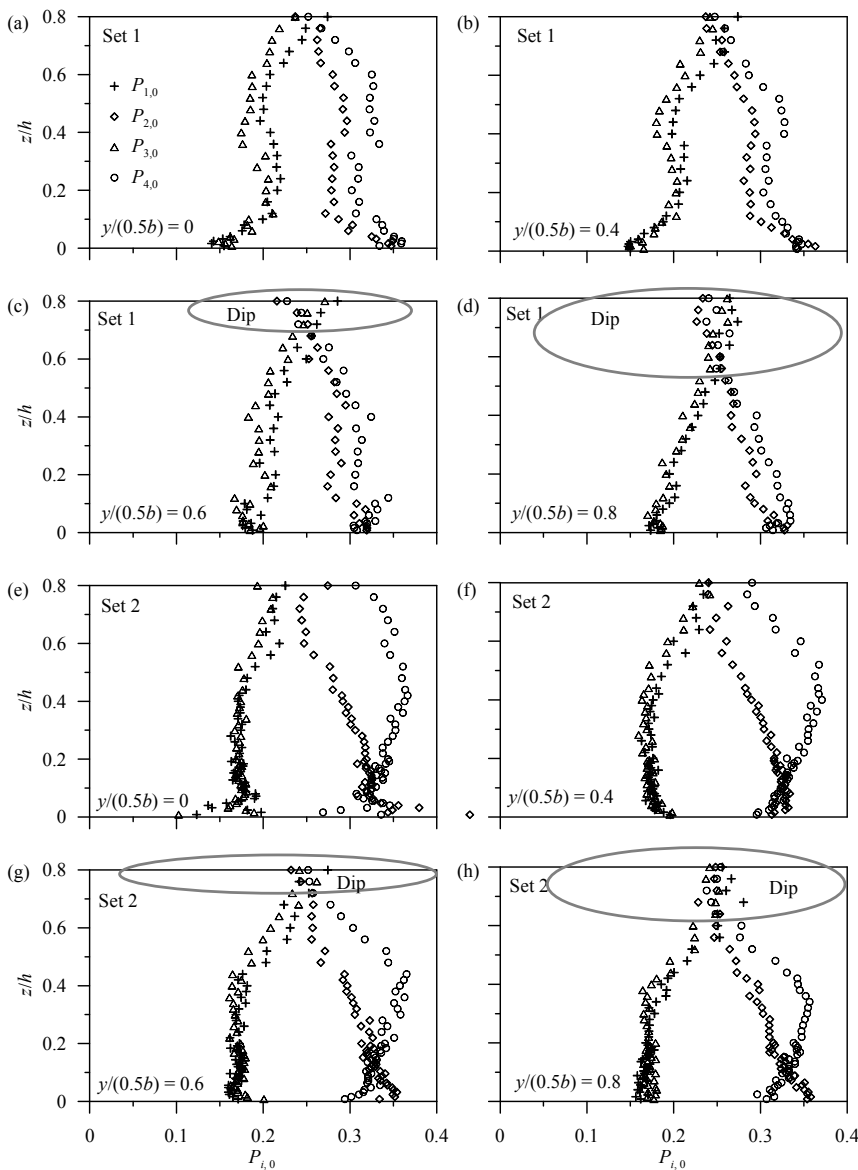

Fig. 7. Vertical distribution of probability of the bursting events $P_{i, H}$ for $H=0$ for (a-d) hydraulically smooth and (e-h) hydraulically rough bed at $y /(0.5 b)=0,0.4,0.6$ and 0.8 .
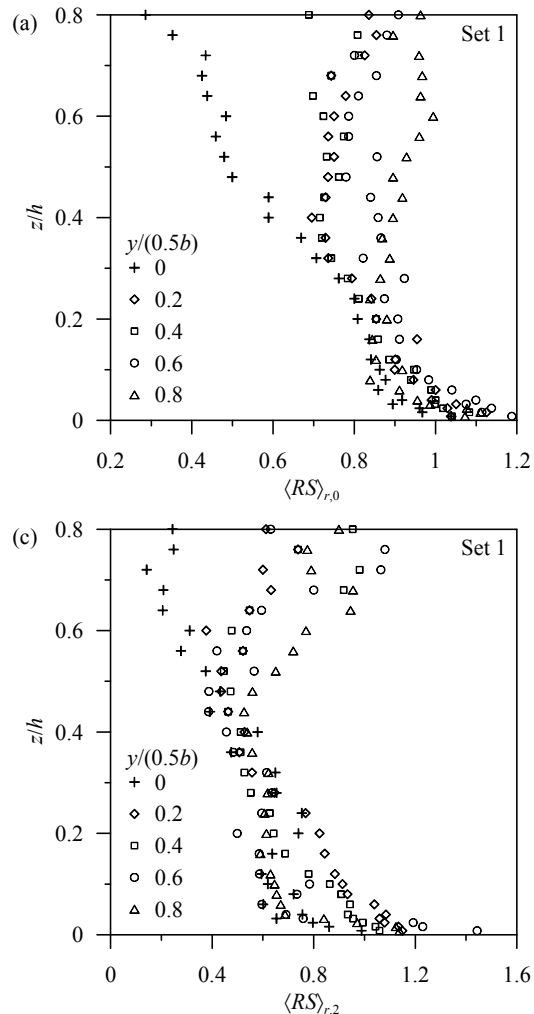
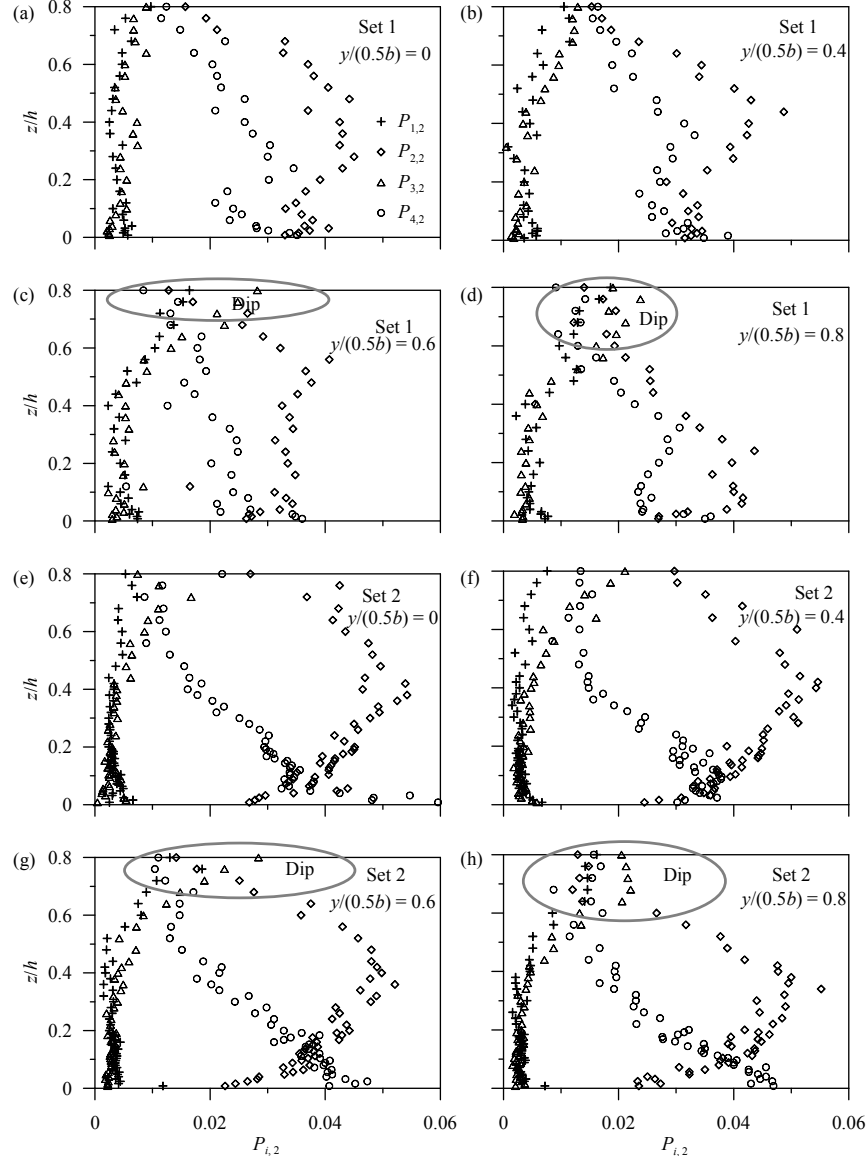

Fig. 8. Vertical distribution of probability of the bursting events $P_{i, H}$ for $H=2$ for (a-d) hydraulically smooth and (e-h) hydraulically rough bed at $y /(0.5 b)=0,0.4,0.6$ and 0.8 .
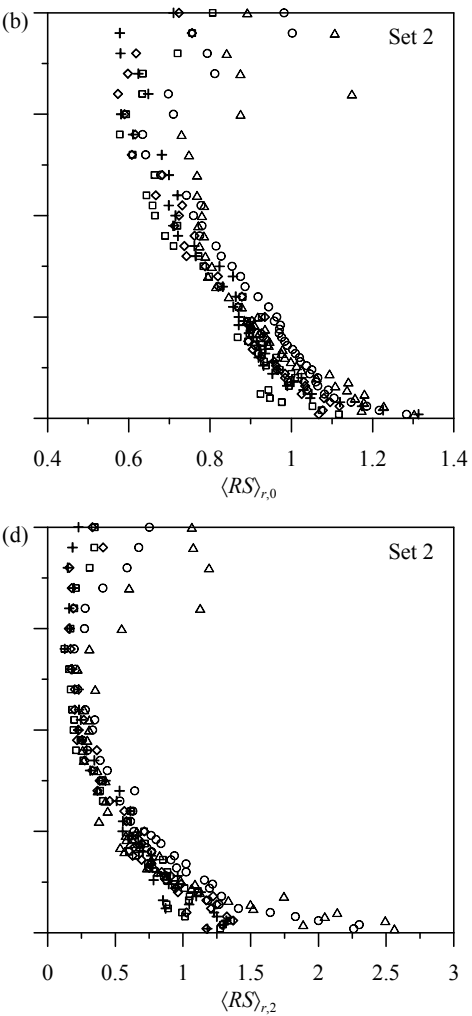

Fig. 9. Vertical distributions of the ratio of sweeps and ejections $\langle R S\rangle_{r, H}$ for (a-b) at $H=0$ and (c-d) at $H=2$, for hydraulically smooth and rough beds at $y /(0.5 b)=0,0.4,0.6$ and 0.8 . 

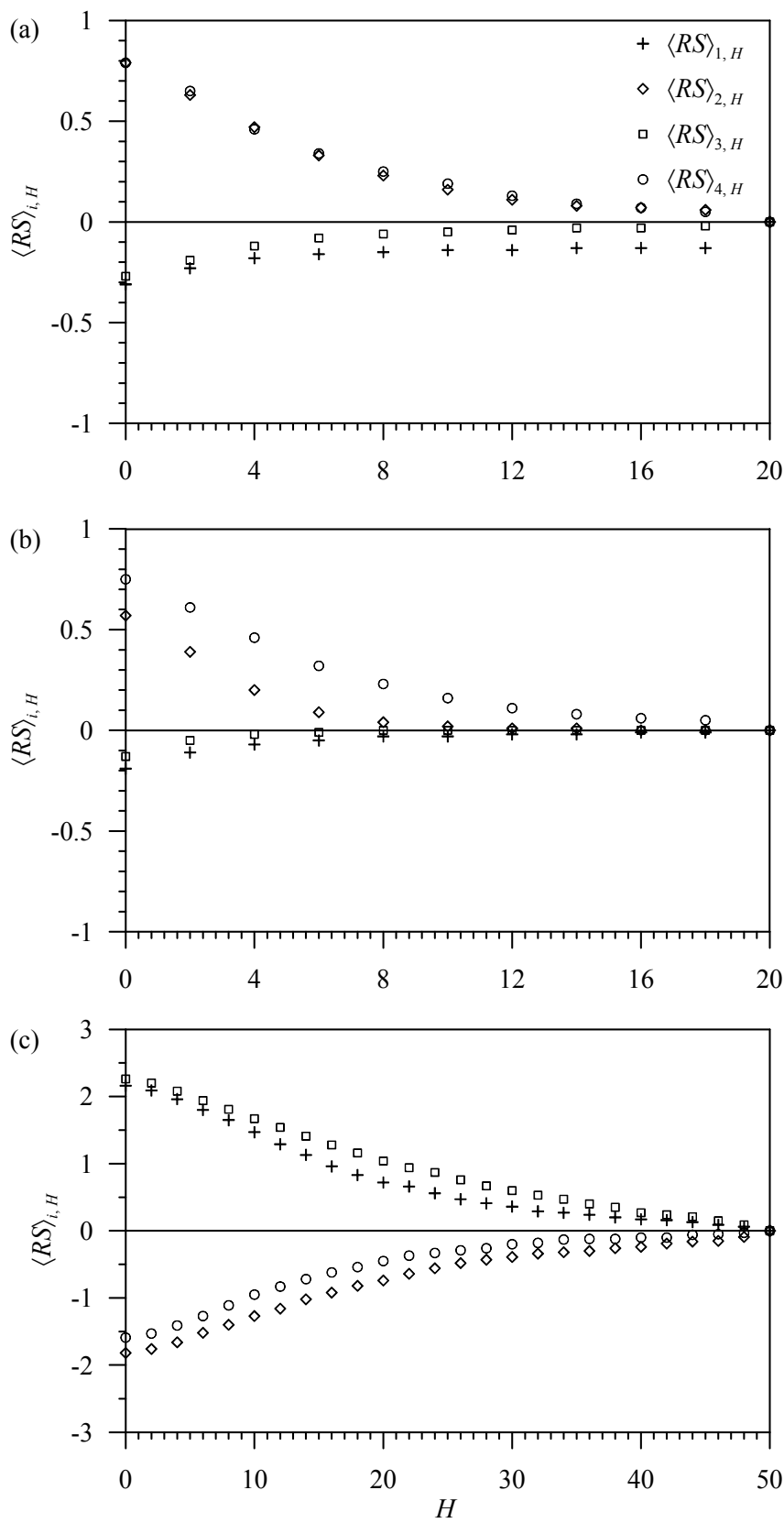

Fig. 10. Variations of $\langle R S\rangle_{i, H}$ with different hole size $H$ for (a) hydraulically smooth bed at $z / h=0.01$, (b) hydraulically rough bed at $z / h=0.01$, and (c) hydraulically rough bed at $z / h=0.6$ at for $y /(0.5 b)=0.8$.

ejections are more than that of inward and outward interactions. In the vicinity of the rough bed, probability of occurring sweeps is the maximum. At the near bed, the probability of sweeps and ejections are basically depends on the bed characteristics i.e., whether smooth or rough. However, as the vertical distance increases, probability of occurring ejections becomes more. The profiles are almost similar for both the rough and smooth beds up to $60 \%$ of total depth irrespective of the $y /(0.5 b)$-values. Above $60 \%$ of the flow depth, probability of occurring the ejections is the maximum for $y /(0.5 b) \leq 0.4$. For the data plots $y /(0.5 b)=0.6$ and 0.8 , the probability of the occurrence of inward and outward interactions become more than that of ejections and sweeps above $60 \%$ of the flow depth for smooth and rough beds. It is therefore observed that within the dip, the probability of the occurrence of inward and outward interactions are more than that of ejections and sweeps. Figs. 8(a-d) and Figs. $8(\mathrm{e}-\mathrm{h})$ present the vertical profiles of $P_{i, H}$ for hydraulically smooth and rough beds respectively for the hole size, $H$ $=2$. It is evident from the figures that the profiles are similar to that with $H=0$ with difference in values.

Figs. $9(\mathrm{a}-\mathrm{b})$ and $(\mathrm{c}-\mathrm{d})$ show the vertical distributions of the ratio of sweeps and ejections $\langle R S\rangle_{r}$ for hydraulically smooth and rough beds at $y /(0.5 b)=0,0.4,0.6$ and 0.8 for $H=0$ and $H=2$, respectively. It is observed from the figures that for both the hole sizes and bed conditions, the data plot collapse on a single band up to $z / h=0.4$. However, for the smooth bed the $\langle R S\rangle_{r}$ deviates from the band. The values of $\langle R S\rangle_{r}$ increase with increase in $y /(0.5 b)$-values. On the other hand, for the rough bed, the data plot follows the single bed even up to 0.6h. Above $0.6 h$, the $\langle R S\rangle_{r}$-values increase with increase in $y /(0.5 b)$-values. At $y /(0.5 b)=0.8$, the value of $\langle R S\rangle_{r}$ is maximum for both the beds.

Figs. $10(\mathrm{a}-\mathrm{c})$ show the variations of the conditional Reynolds shear stress with respect to hole size $H$ at three different conditions: close to the smooth bed $(0.1 h)$, close to the rough bed $(0.1 h)$ and within the dip $(0.7 h)$ for smooth bed at $y /(0.5 b)$ $=0.8$. The overall trend of the events with respect to the hole size is similar for all the cases. With increase in hole size their effect becomes less and finally at larger values of $H$ they become zero. It was also observed that, closest to the rough bed, sweep becomes the governing mechanism. Interestingly, for the flow zone within the dip, all the bursting events change their signs.

\section{CONCLUSION}

The turbulence characteristics over hydraulically smooth and rough beds are studied experimentally. A detailed study of the turbulent bursting has been portrayed within the dip in a narrow open channel. Near the sidewall (corner) of the flume, the velocity-dip was observed above nearly $60 \%$ of the flow depth. Within the dip, the maximum velocity was observed below the free-surface (Fig. 2 and 4), the nondimensional Reynolds shear stress exhibits very small values and its sign changes from positive to negative (Fig. 3). The quadrant plots for the turbulent bursting shows that all the bursting events change their signs within the dip due to the changes of the sign of nondimensional Reynolds shear stress (Figs. 5-6). In the vicinity of the bed, probability of the occurrence of sweeps and ejections are more than that of inward and outward interactions. On the other hand, within the dip, the probability of the occurrence of the outward and inward interactions become more than that of sweeps and ejections (Figs. 7-8). It was also observed that the data plots below the dip, follow almost similar trends for flows over smooth and rough beds. The relative dominance of the bursting events is shown by the vertical distributions of the ratio of sweeps and ejections (Fig. 9). The variation of busting events with respect to hole size suggested that all the events are quite effective at lower hole size, while their contributions become feeble with an increase in hole size irrespective of the flow conditions. The changes of the signs of the bursting events are also observed within the dip (Fig. 10).

Acknowledgements. The author is grateful to the anonymous reviewers for their constructive comments and suggestions which greatly improved the manuscript. 


\section{REFERENCES}

Absi, R., 2011. An ordinary differential equation for velocity distribution and dip-phenomenon in open channel flows. J. Hydraul. Res., 49, 1, 82-89.

Auel, C., Albayrak, I., Boes, R., 2014. Turbulence characteristics in supercritical open channel flows: effects of Froude number and aspect ratio. J. Hydraul. Eng., 140, 4, 04014004.

Cea, L, Puertas, J., Pena, L., 2007. Velocity measurements on highly turbulent free surface flow using ADV. Exp. Fluids, 42, 3, 333-348.

Chow, V.T., 1959. Open Channel Hydraulics. McGraw-Hill Book Company, New York.

Corino, E.R., Brodkey, R.S., 1969. A visual investigation of the wall region in turbulent flow. J. Fluid Mech., 37, 1, 1-30.

Dey, S., 2014. Fluvial Hydrodynamics: Hydrodynamic and Sediment Transport Phenomena. Springer-Verlag, Berlin, Heidelberg.

Dey, S., Nath, T.K., 2010. Turbulence characteristics in flows subjected to boundary injection and suction. J. Eng. Mech., ASCE, 136, 7, 877-888.

Dey, S., Das, R., 2012. Gravel-bed hydrodynamics: Doubleaveraging approach. J. Hydraul. Eng., 138, 8, 707-725.

Franca, M.J., Lemmin, U., 2009. The simultaneous occurrence of logarithmic and S-shaped velocity profiles in gravel-bed river flows. Arch. Hydro-Eng. and Environ. Mech., 56, 1-2, 29-41.

Grass, A.J., 1971. Structural features of turbulent flow over smooth and rough boundaries. J. Fluid Mech., 50, 2, 233255.

Goring, D., Nikora, V.I., 2002. Despiking acoustic Doppler velocimeter data. J. Hydraul. Eng., 128, 1, 117-126.

Guo, J., Julien, P.Y., 2008. Application of the modified logwake law in open-channels. J. Appl. Fluid Mech., 1, 2, 1723.

Kline, S.J., Reynolds, W.C., Schraub, F.A., Runstadler, P.W., 1967. The structure of turbulent boundary layers. J. Fluid Mech., 30, 741-773.

MacVicar, B.J., Dilling, S., Lacey, R.W.J., Hipel, K., 2014. A quality analysis of the Vectrino II instrument using a new open-source MATLAB toolbox and 2D ARMA models to detect and replace spikes. In: Proc. Conf. River Flow 2014, Lausanne, Switzerland.

Mignot, E., Barthelemy, E., Hurther, D., 2009. Doubleaveraging analysis and local flow characterization of nearbed turbulence in gravel-bed channel flows. J. Fluid Mech., 618, 279-303.

Najafabadi, E.F., Afzalimehr, H., Sui, J., 2015. Turbulence characteristics of favorable pressure gradient flows in gravel-bed channel with vegetated walls. J. Hydrol. Hydromech., 63, 2, 154-163.
Nezu, I., Nakagawa, H., 1993. Turbulence in Open-Channel Flows. Balkema, Rotterdam.

Parsheh, M., Sotiropoulos, F., Port e-Agel, F., 2010. Estimation of power spectra of acoustic-Doppler velocimetry data contaminated with intermittent spikes. J. Hydraul. Eng., 136, 6, 368-378.

Rodríguez, J.F., García, M.H., 2008. Laboratory measurements of 3-D flow patterns and turbulence in straight open channel with rough bed. J. Hydraul. Res., 46, 454-465.

Sarma, K.V.N., Prasad, B.V.R., Sarma, A.K., 2000. Detailed study of binary law for open channels. J. Hydraul. Eng., 126, $3,210-214$.

Tracy, H.J., Lester, C.M., 1961. Resistance coefficients and velocity distribution in smooth rectangular channel. U.S. Geological Survey (USGS) Water-supply paper 1592-A, USGS, Washington, D.C., A1-A18.

Vanoni, V.A., 1941. Velocity distribution in open channels. Civil Eng., 11, 6, 356-357.

Willmarth, W.W., Lu, S.S., 1972. Structure of the Reynolds stress near the wall. J. Fluid Mech., 55, 65-92.

Wright, J.D., Baas, J.H., 2013. Despiking ultrasonic Doppler velocity-profiling data. J. Sedimentary Res. (Research Methods), 83, 11, 954-961.

Yang, S.Q., Tan, S.K., Lim, S.Y., 2004. Velocity distribution and dip-phenomenon in smooth uniform open channel flows. J. Hydraul. Eng., 130, 12, 1179-1186.

\section{NOMENCLATURE}

$b \quad=\quad$ width of the flume $(\mathrm{L})$

$b_{i k} \quad=\quad$ Reynolds stress anisotropy tensor $(-)$

$d_{50}=$ median diameter of gravels $(\mathrm{L})$

$\mathrm{Fr} \quad=\quad$ Froude number $(-)$

$\mathrm{Re} \quad=\quad$ Reynolds number $(-)$

$h \quad=\quad$ flow depth $(\mathrm{L})$

$\langle R S\rangle_{i, H}=$ Reynolds shear stress fractional contribution $(-)$

$\left\langle R S_{r}\right\rangle_{i, H}=\langle R S\rangle_{4, H} /\langle R S\rangle_{2, H}(-)$

$R_{*} \quad=\quad$ particle Reynolds number $\left(=d_{50} u_{*} / v\right)(-)$

$u=$ instantaneous streamwise velocity component $\left(\mathrm{LT}^{-1}\right)$

$u^{\prime}=$ instantaneous fluctuations of $u\left(\mathrm{LT}^{-1}\right)$

$w \quad=\quad$ instantaneous vertical velocity component $\left(\mathrm{LT}^{-1}\right)$

$w^{\prime} \quad=\quad$ fluctuations of $w\left(\mathrm{LT}^{-1}\right)$

$z \quad=\quad$ vertical distance $(\mathrm{L})$

$\rho \quad=\quad$ mass density of fluid $\left(\mathrm{ML}^{-3}\right)$

$v \quad=$ kinematic viscosity of fluid $\left(\mathrm{L}^{2} \mathrm{~T}^{-1}\right)$

Received 9 October 2015 Accepted 12 February 2016

Note: Colour version of Figures can be found in the web version of this article. 